\title{
Foxj2 overexpression is associated with poor prognosis, progression, and metastasis in nasopharyngeal carcinoma
}

This article was published in the following Dove Press journal:

OncoTargets and Therapy

24 July 2017

Number of times this article has been viewed

\author{
Ying Shan ${ }^{1, *}$ \\ Tao Chang ${ }^{2, *}$ \\ Si Shi' \\ Mingming Tang ${ }^{3}$ \\ Lili Bao' \\ $\mathrm{Li} \mathrm{Li'}$ \\ Bo You' \\ Yiwen You'
}

'Department of Otorhinolaryngology, Head and Neck Surgery, Affiliated Hospital of Nantong University,

Nantong, ${ }^{2}$ Department of

Otorhinolaryngology, Head and Neck Surgery, The First People's Hospital of Wujiang, Wujiang, ${ }^{3}$ Department of Otorhinolaryngology, Head and Neck Surgery, Affiliated Cancer Hospital of Nantong University, Nantong, Jiangsu Province, People's Republic of China

*These authors contributed equally to this work
Correspondence: Yiwen You; Bo You Department of Otorhinolaryngology, Head and Neck Surgery, Affiliated Hospital of Nantong University, 20 Xisi Road, Nantong, Jiangsu 22600I, People's Republic of China

$\mathrm{Tel} / \mathrm{fax}+86$ I35 8522 9333;

$+8615851358688$

Email youyiwen_nantong@163.com; youbo19891014@163.com

\begin{abstract}
Foxj2, a novel member of Forkhead box family, has been reported to play an important role in tumorigenesis, progression, and metastasis of certain cancers. However, the expression status and effects of Foxj2 on nasopharyngeal carcinoma (NPC) progression and metastasis remain debated. In this study, we first examined the expression of Foxj2 in NPC by immunohistochemistry and Western blotting analysis. We confirmed significantly elevated expression of Foxj 2 in NPC tissues and cell lines. Next, the relationships between Foxj2 expression levels and the clinicopathological factors were investigated. Its expression level correlated with T-classification $(P=0.026)$, distant metastasis $(P=0.004)$, and clinical stage $(P=0.029)$. In addition, high expression of Foxj 2 was associated with poor prognosis in NPC patients. The effects of Foxj 2 on cell proliferation and migration were explored by RNA interference (RNAi) with CCK-8 assay, cell cycle analyses, wound healing, and transwell assay. In conclusion, our data indicate that Foxj 2 upregulation promotes the progression and migration of NPC. It makes Foxj2 serve as a potential therapeutic target for the treatment of NPC.
\end{abstract}

Keywords: Foxj2, nasopharyngeal carcinoma, prognosis, progression, migration

\section{Introduction}

Nasopharyngeal carcinoma (NPC) is the most common head and neck cancer with dramatically different geographical and ethnic distribution patterns. ${ }^{1}$ It has high rates of local recurrence and distant metastasis, and $90 \%$ of patients show cervical lymph node metastasis at the time of diagnosis. ${ }^{2}$ Therefore, understanding the mechanisms associated with NPC progression, metastasis, and prognosis is crucial.

Forkhead box (Fox) family, which includes more than 80 members of transcription factors, share a common forkhead DNA-binding domain, termed the "fork-head" or "winged-helix" motif. ${ }^{3}$ Most of the members of the Fox family have been reported to be widely present in different organs of different species ranging from yeast to humans and play extraordinarily diverse roles in embryonic development by regulating the expression of different sets of target genes. ${ }^{4}$ The role played by these transcription factors of the Fox family is important for a wide spectrum of biological processes such as signal transduction, metabolism, development, cell differentiation, proliferation, apoptosis, migration, invasion, and even longevity. ${ }^{5-11}$ Currently, mounting evidence suggests that Fox family members are abnormally expressed in many cancers, including NPC, and are involved in a variety of cellular processes that are abnormal in cancer cells, such as proliferation, differentiation, adhesion, migration, and invasion. ${ }^{12-17}$ Forkhead box J2 (Foxj2), a member of this family formerly named as FHX, has dual DNA-binding 
specificity. ${ }^{18}$ Previous studies have shown its biological importance in mouse embryogenesis and tumorigenesis. ${ }^{19,20}$ The expression patterns of Foxj2 have been reported to be aberrant in a variety of cancers, such as breast cancer, extrahepatic cholangiocarcinoma, glioma, non-small cell lung cancer. ${ }^{21-24}$ In addition, it is reported that Foxj2 appears to be involved in regulating the progression and metastasis of the cancers, as well as aiding in tumorigenesis. ${ }^{21,24}$ However, its possible roles in NPC are still unknown.

The purpose of this article was demonstrating the expression levels and roles of Foxj 2 in the prognosis, progression, and metastasis of NPC. We first determined the expression of Foxj 2 in NPC. Then, its associations with clinical and pathologic factors were evaluated. Furthermore, we explored the biological functions, such as proliferation and migration of Foxj2 in NPC, by silencing its expression. All the data suggested that Foxj 2 might be a positive regulator in NPC progression.

\section{Materials and methods}

\section{Tissue specimens}

A total of 57 NPC sections from patients who underwent biopsy at the Affiliated Hospital of Nantong University, China, were fixed in formalin and embedded in paraffin for histopathologic diagnosis and immunohistochemistry (IHC). Noncancerous nasopharyngeal tissues were collected from patients with clinical symptoms suggestive of NPC but ruled out by biopsy. Before biopsy, none of the patients with newly diagnosed NPC had received any antitumor therapy. The main clinical and pathologic characteristics are listed in Table 1. The fresh tissues were frozen in liquid nitrogen immediately after biopsy and stored at $-80^{\circ} \mathrm{C}$ until use. This study was approved by the ethics committee of the Affiliated Hospital of Nantong University and all participants gave informed consent.

\section{IHC staining}

IHC analysis was carried out as previously reported. ${ }^{25,26}$ The primary antibody used was ab22857 against Foxj2 (1:50;

Table I Sequences of siRNAs targeting Foxj2

\begin{tabular}{ll}
\hline siRNA names & Sequences $\left(\mathbf{5}^{\prime} \mathbf{- 3}^{\prime} \mathbf{)}\right.$ \\
\hline Foxj2_siRI & Sense: CGAACAACUACUACAUGUAdTdT \\
& Antisense: UACAUGUAGUAGUUGUUCGdTdT \\
Foxj2_siR2 & Sense: CCUUCUGACUGGUGCUCUAdTdT \\
& Antisense: UAGAGCACCAGUCAGAAGGdTdT \\
Foxj2_siR3 & Sense: GGCACAACCUUUCUCUCAAdTdT \\
& Antisense: UUGAGAGAAAGGUUGUGCCdTdT \\
Foxj2_siR4 & Sense: GGUCCUUCCGCAACCUCUAdTdT \\
& Antisense: UAGAGGUUGCGGAAGGACCdTdT \\
\hline
\end{tabular}

Abbreviations: siRNA, small interfering RNA; siR, siRNA.
Santa Cruz Biotechnology Inc, Dallas, TX, USA) and slides were incubated overnight at $4^{\circ} \mathrm{C}$. Negative control slides were processed in parallel with nonspecific immunoglobulin $G$ (IgG) (Sigma Chemical Co, St Louis, MO, USA) at the same concentration as the primary antibody. Sections were then washed and treated with horseradish peroxidase-conjugated goat anti-rabbit antibody (DakoCytomation, Carpinteria, CA, USA) for 15 min. For assessment of Foxj2, 2 blinded pathologists evaluated the immunostaining (including the staining intensity and percentage of positive cells). The intensity was estimated as follows: strong staining (3), moderate staining (2), weak staining (1), or negative staining (0). The scale of positive cells was graded as follows: $0 \%$ of positive cells ( 0 ), $1 \%-10 \%$ of positive cells (1), 10\%-50\% of positive cells (2), $51 \%-75 \%$ of positive cells (3), $76 \%-100 \%$ of positive cells (4). The results of intensity and extent were added as described. ${ }^{24}$ The samples with a final score of 0 were considered to represent negative expression; $2-3$ indicated weak expression; 4-5 was considered moderate expression; and 6-7 was indicative of strong expression. For statistical analysis, scores of $0-3$ were regarded as Foxj2 negative or weak expression and 4-7 were determined as overexpression.

\section{Western blot analysis}

Western blot analysis was performed as described previously. ${ }^{25,26}$ The primary antibodies used for Western blot analysis were as follows: Foxj2 polyclonal antibody $(1: 1,000$; Santa Cruz Biotechnology) and $\beta$-actin polyclonal antibody (1:2,000; Santa Cruz Biotechnology).

\section{Cell cultures}

The process for obtaining the conditioned medium from the cells was the same as that in our previous study. ${ }^{27}$ In brief, NPC cell lines (CNE-1, CNE-2, 5-8F, and 6-10B) were maintained in RPMI 1640 (Gibco BRL, Grand Island, NY, USA) containing additionally $10 \%$ fetal bovine serum (FBS; Gibco BRL), while NP69 (normal nasopharyngeal epithelial cell line) was cultured in Keratinocyte-SFM (Invitrogen, Carlsbad, CA, USA). All the cells were incubated at $37^{\circ} \mathrm{C}$ in $5 \% \mathrm{CO}_{2}$ incubator.

\section{Small interfering RNA (siRNA) transfection}

The Foxj2 siRNA and negative control siRNA were designed and obtained from Biomics Biotechnologies Co, Ltd (Nantong, China). The sequences of siRNAs are shown in Table 1. Before transfection, cells were plated and allowed to grow to $30 \%-50 \%$ confluence in 6 -well plates. According to 
the manufacturer's suggestion, the siRNAs were transfected with Lipofectamine 2000 (Invitrogen).

\section{Cell proliferation assay}

The cell proliferation was assessed using the cell counting kit-8 (CCK-8) assay. Cells transfected with Foxj2 or control siRNA were seeded in 96-well plates (20,000 cells per well) and grown overnight. At time points of 0, 6, 12, 24, 36, and $48 \mathrm{~h}, 10 \mu \mathrm{L}$ per well CCK-8 solution was added and incubated for $2 \mathrm{~h}$ at $37^{\circ} \mathrm{C}$. The absorbance was measured at $450 \mathrm{~nm}$ using a microplate reader.

\section{Cell cycle analyses}

For cell cycle analysis, cells transfected with Foxj 2 or control siRNA were fixed and incubated with RNase A. Subsequently, the cells were stained with Cell Cycle Detection Kit (KeyGene, Wageningen, the Netherlands) according to the manufacturer's instructions. Cells were analyzed using a BD FACScan flow cytometer (Becton Dickinson, San Jose, CA, USA) and Cell Quest software. All samples were assayed in triplicate.

\section{Transwell assays}

Cell migration was measured using cell culture inserts (24-well type, $8 \mu \mathrm{m}$ pore size; Corning Inc, Corning, NY, USA). Subsequently, $1 \times 10^{5}$ cells transfected with Foxj 2 or control siRNA were added into the upper chambers with the serum-free RPMI 1640 medium, while the lower chambers were filled with $500 \mu \mathrm{L}$ complete RPMI 1640. After $16 \mathrm{~h}$ of incubation, the cells that had migrated to the undersurface of the membrane were fixed in $100 \%$ methanol and stained with crystal violet. Cells were visualized and 10 random fields were counted under a microscope.

\section{Wound-healing assay}

Cells transfected with Foxj2 or control siRNA were plated in 6-well plates. After the cells reached $80 \%$ confluence, scratches were made using a $100 \mu \mathrm{L}$ pipette tip. Wound healing was observed and the migration distance was imaged at different time points.

\section{Calculation and statistical analysis}

All the quantified data from 3 independent experiments are expressed as the mean values \pm SDs and analyzed using SPSS17.0 statistical software. The $\chi^{2}$ test was used to determine the significance of Foxj 2 expression and the clinicopathological features of NPC. Survival data were calculated by the Kaplan-Meier method and compared by the log-rank test. Statistical significance was determined by the 2-tailed
Student's $t$-test for 2 groups and 1-way analysis of variance (ANOVA) for more than 2 groups. The value of $P<0.05$ was considered statistically significant.

\section{Results}

\section{Foxj2 was overexpressed in NPC}

In order to characterize the role of Foxj2 in NPC, we first detected the expression and subcellular localization of Foxj 2 in NPC tissues by IHC analysis. We found that Foxj 2 had higher expression in NPC $(34 / 57,59.65 \%)$ than in nontumor tissues (4/26, 15.38\%) (Figure 1A). A combination of nuclear and cytoplasmic positive staining was observed in most of the NPC samples (Figure 1A). The results of IHC staining are summarized in Table 2. Western blotting was further performed to assess the expression level of Foxj2 (Figure 1B and C). In agreement with these data, Foxj2 was found to be markedly overexpressed in NPC tissues (Figure $1 \mathrm{~B}$ and $\mathrm{C}$ ). These data raised the possibility that high levels of Foxj2 might be a potential biomarker for NPC.

\section{Correlation between Foxj2 expression and clinicopathological parameters}

We further explored the association of Foxj2 expression with clinicopathologic variables in NPC. The clinicopathological data of Foxj 2 expression are summarized in Table 2. As shown, high Foxj2 expression was significantly associated with T-classification ( $P=0.026)$, distant metastasis $(P=0.004)$, and clinical stage $(P=0.029)$.

\section{Overexpression of Foxj2 was indicative of poor survival for NPC patients}

To know whether the overexpression of Foxj 2 was correlated with survival, Kaplan-Meier analysis was performed to study the prognostic significance of Foxj2. The survival curves indicated that patients with Foxj 2 overexpression had lower overall survival rate than those with negative expression (Figure 2) $(P<0.001)$.

Moreover, univariate and multivariate analyses showed that NPC patients' overall survival rate were significantly related to Foxj2 expression and that Foxj2 expression $(P=0.031)$ was an independent prognostic factor in NPC patients (Tables 3 and 4). These results strengthen our conjecture that there may be a correlation between the overexpression of Foxj2 and clinical survival in NPC patients.

\section{Expression of Foxj2 in NPC cells}

To further assess the biological role of Foxj2 in NPC, we carried out cell-based experiments. First, the expression of Foxj2 in NPC cell lines was investigated. As shown 


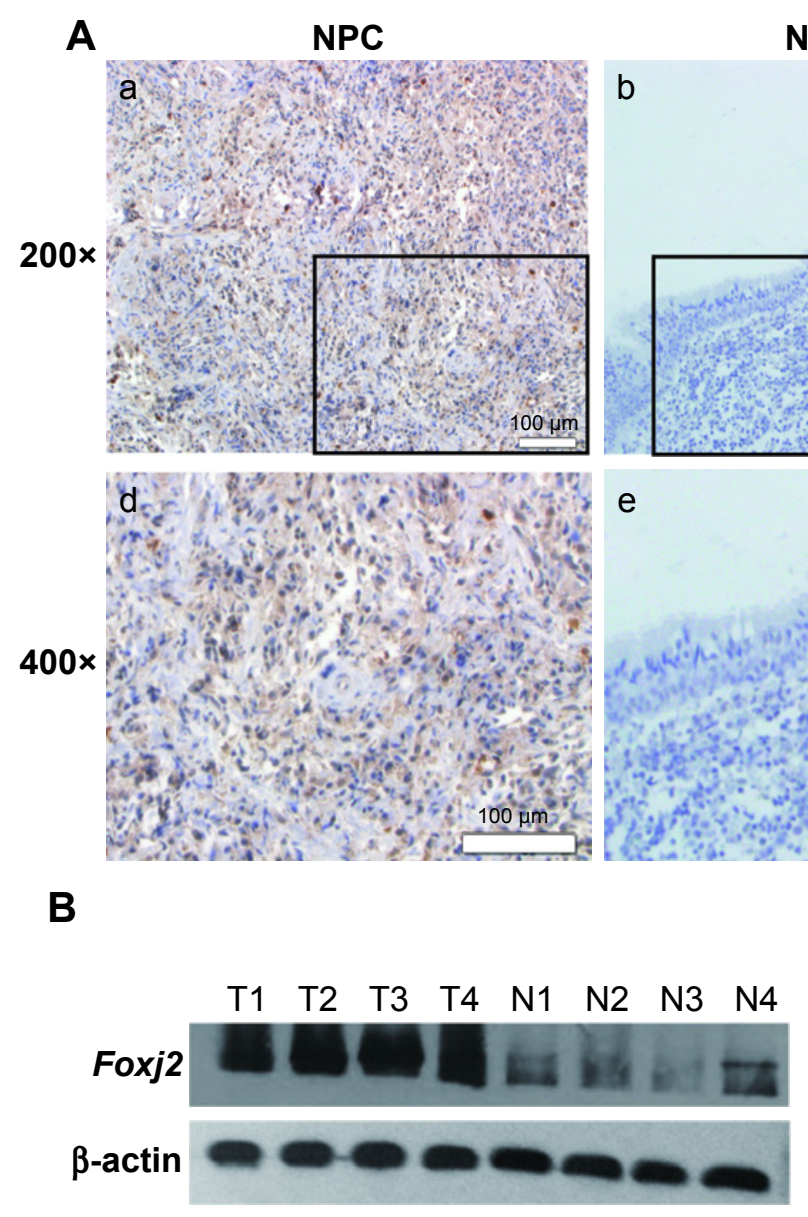

Normal
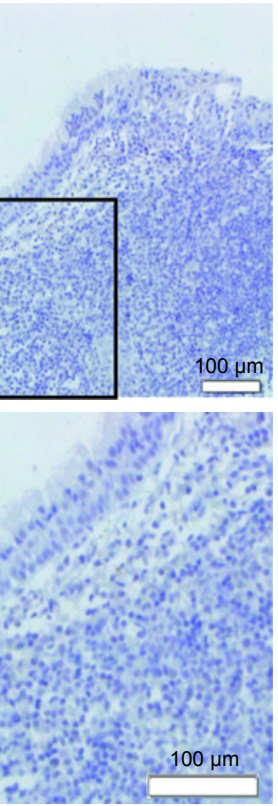

C

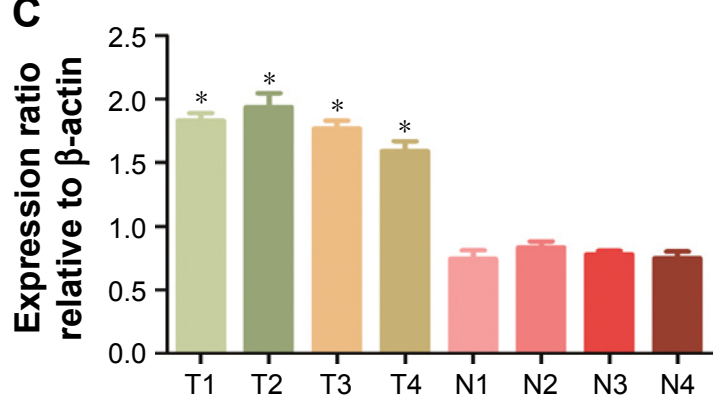

Figure I Expression pattern and prognosis role of Foxj2 in NPC tissues.

Notes: (A) Immunohistochemistry analysis (I:50, ab22857) of Foxj2 expression in NPC tissues. Original magnifications: $\times 200$ (a-c); $\times 400$ (d-f) (B) Protein levels of Foxj2 in NPC (ie, tumor tissue T) and noncancerous nasopharyngeal tissues (labeled "N") by Western blotting. (C) Quantitative results of Western blotting. $\beta$-actin was used as a loading control. The same experiment was repeated at least 3 times. $* P<0.05$. Data are presented as mean \pm SD.

Abbreviation: NPC, nasopharyngeal carcinoma.

in Figure $3 \mathrm{~A}$ and B, Foxj2 were overexpressed in all 4 kinds of NPC cell lines compared with the normal nasopharyngeal epithelial cell line NP69 (Figure 3A and B). To clarify the function of Foxj2 clearly, 4 siRNAs were designed and transfected into CNE-2 cells to knock down the Foxj2 expression. Foxj2-si3 showed the highest knockdown efficiency, and the expression level was inhibited by Foxj2-si3 up to $56.6 \%$ (Figure 3C and D).

\section{Foxj2 siRNA inhibits cell proliferation}

As IHC analysis showed overexpression of Foxj2 in T3-T4 tissues versus $\mathrm{T} 1-\mathrm{T} 2$, we hypothesized that Foxj 2 was closely related to NPC proliferation (Figure 4A). Next, CCK-8 and flow cytometry assays were carried out to assess the cell proliferation function of Foxj2 on NPC. CCK-8 assays showed that after downregulating the Foxj2 expression, the CNE-2 cells showed a significant decrease of the cell proliferation rate (Figure 4B and C). Consistent with these results, flow cytometry analysis revealed that CNE-2 cells accumulated in the $\mathrm{G} 0 / \mathrm{G} 1$ phase (from $42.57 \%$ to $58.78 \%$ ), whereas those in the $\mathrm{S}$ phase decreased (from 39.01\% to $30.79 \%$ ) after downregulating Foxj2 expression with Foxj2-si3 (Figure 4D), suggesting that Foxj2 could promote accumulation of the cells in the $\mathrm{S}$ phase and thus promote the cell growth. These results suggested that Foxj 2 might play a crucial role in regulation of NPC cell proliferation.

\section{Foxj2 siRNA inhibits cell migration}

As IHC analysis showed that Foxj2 overexpression is significantly associated with distant metastasis in NPC 
Table 2 The association between Foxj2 expression and clinicopathological features in 57 NPC patients

\begin{tabular}{|c|c|c|c|c|}
\hline \multirow[t]{2}{*}{$\begin{array}{l}\text { Clinicopathological } \\
\text { parameters }\end{array}$} & \multirow[t]{2}{*}{ Total } & \multicolumn{2}{|c|}{$\begin{array}{l}\text { Foxj2 } \\
\text { expression, } n\end{array}$} & \multirow[t]{2}{*}{$P$-value } \\
\hline & & Low & High & \\
\hline \multicolumn{5}{|l|}{ Gender } \\
\hline Male & 41 & 17 & 24 & 1.000 \\
\hline Female & 16 & 6 & 10 & \\
\hline \multicolumn{5}{|l|}{ Age, years } \\
\hline$<50$ & 20 & 8 & 12 & 1.000 \\
\hline$\geq 50$ & 37 & 15 & 22 & \\
\hline \multicolumn{5}{|l|}{ T-classification } \\
\hline TI-T2 & 37 & 19 & 18 & $0.026 *$ \\
\hline T3-T4 & 20 & 4 & 16 & \\
\hline \multicolumn{5}{|l|}{ Lymph node metastasis } \\
\hline NO-NI & 38 & 13 & 25 & 0.253 \\
\hline N2-N3 & 19 & 10 & 9 & \\
\hline \multicolumn{5}{|l|}{ Distant metastasis } \\
\hline No & 43 & 22 & 21 & $0.004 *$ \\
\hline Yes & 14 & 1 & 13 & \\
\hline \multicolumn{5}{|l|}{ TNM clinical stage } \\
\hline I-II & 24 & 14 & 10 & $0.029 *$ \\
\hline III-IV & 33 & 9 & 24 & \\
\hline
\end{tabular}

Notes: Statistical analyses were performed by the Pearson's $\chi^{2}$ test. $* P<0.05$ was considered significant.

Abbreviations: NPC, nasopharyngeal carcinoma; TNM, tumor-node-metastasis.

(Figure 5A), we investigated the cell migration effects of Foxj2. Wound-healing and transwell assays were carried out to examine the migration impact of Foxj2 knockdown on CNE-2 cells. Interestingly, both the assays suggested that knockdown of Foxj2 significantly inhibited cell migration (Figure 5B-E). These data indicated that downregulation of Foxj2 reduced the migration of CNE-2 cells.

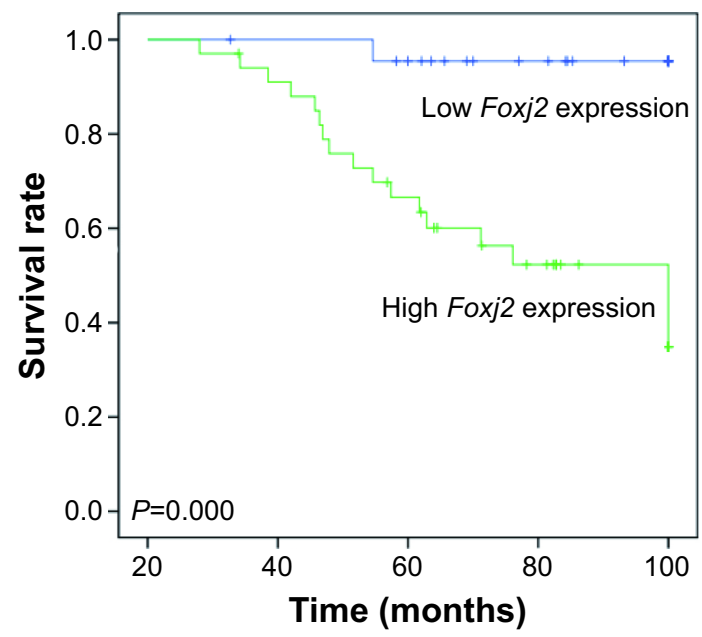

Figure 2 Prognosis role of Foxj2 in NPC.

Notes: Kaplan-Meier survival curves of NPC patients based on Foxj2 expression status; $P<0.05$, log-rank test.

Abbreviation: NPC, nasopharyngeal carcinoma.
Table 3 Survival status and clinicopathological parameters in 57 human NPC tissues

\begin{tabular}{|c|c|c|c|c|}
\hline \multirow[t]{2}{*}{$\begin{array}{l}\text { Clinicopathological } \\
\text { parameters }\end{array}$} & \multirow[t]{2}{*}{ Total } & \multicolumn{2}{|c|}{$\begin{array}{l}\text { Survival status, } \\
\text { n }\end{array}$} & \multirow[t]{2}{*}{$P$-value } \\
\hline & & Alive & Dead & \\
\hline \multicolumn{5}{|l|}{ Gender } \\
\hline Male & 41 & 29 & 12 & 0.545 \\
\hline Female & 16 & 10 & 6 & \\
\hline \multicolumn{5}{|l|}{ Age, years } \\
\hline$<50$ & 20 & 12 & 8 & 0.377 \\
\hline$\geq 50$ & 37 & 27 & 10 & \\
\hline \multicolumn{5}{|l|}{ T-classification } \\
\hline TI-T2 & 37 & 29 & 8 & $0.039 *$ \\
\hline T3-T4 & 20 & 10 & 10 & \\
\hline \multicolumn{5}{|l|}{ Lymph node metastasis } \\
\hline No-NI & 38 & 23 & 15 & 0.081 \\
\hline N2-N3 & 19 & 16 & 3 & \\
\hline \multicolumn{5}{|l|}{ Distant metastasis } \\
\hline No & 43 & 36 & 7 & $0.000 *$ \\
\hline Yes & 14 & 3 & 11 & \\
\hline \multicolumn{5}{|l|}{ TNM clinical stage } \\
\hline I-II & 24 & 19 & 5 & 0.161 \\
\hline III-IV & 33 & 20 & 13 & \\
\hline \multicolumn{5}{|l|}{ Foxj2 expression } \\
\hline Low & 23 & 22 & I & $0.000 *$ \\
\hline High & 34 & 17 & 17 & \\
\hline
\end{tabular}

Notes: Statistical analyses were performed by the Pearson's $\chi^{2}$ test. $* P<0.05$ was considered significant.

Abbreviations: NPC, nasopharyngeal carcinoma; TNM, tumor-node-metastasis.

\section{Discussion}

NPC is the most common head and neck cancer and continues to be one of the leading causes of cancer-related death worldwide. It has long been acknowledged that gene changes play crucial roles in NPC development and metastasis and affect patients' outcome. Therefore, it is imperative for us to understand the gene changes that govern its progression and enable early diagnosis. This study provides the first evidence that overexpressed Foxj2 might be a potential regulator in NPC progression and migration.

Table 4 Contribution of various potential prognostic factors to survival, determined by Cox regression analysis, in 57 human NPC tissues

\begin{tabular}{llll}
\hline Prognostic factors & $\begin{array}{l}\text { Hazard } \\
\text { ratio }\end{array}$ & P-value & $\begin{array}{l}\mathbf{9 5 \%} \text { confidence } \\
\text { interval }\end{array}$ \\
\hline $\begin{array}{l}\text { T-classification } \\
\text { I-II versus III-IV }\end{array}$ & 2.455 & 0.165 & $0.69 \mathrm{I}-8.72 \mathrm{I}$ \\
$\begin{array}{c}\text { Distant metastasis } \\
\quad \text { M0 versus MI }\end{array}$ & 6.689 & $0.002 *$ & $2.02 \mathrm{I}-22.143$ \\
$\begin{array}{c}\text { TNM clinical stage } \\
\text { I-II versus III-IV }\end{array}$ & 0.275 & 0.123 & $0.053-\mathrm{I} .420$ \\
$\begin{array}{c}\text { Foxj2 expression } \\
\text { Low versus high }\end{array}$ & 0.099 & $0.03 \mathrm{I}$ & $0.012-0.806$ \\
\hline
\end{tabular}

Notes: Statistical analyses were performed by Cox regression analysis. $* P<0.05$ was considered significant.

Abbreviations: NPC, nasopharyngeal carcinoma; TNM, tumor-node-metastasis. 

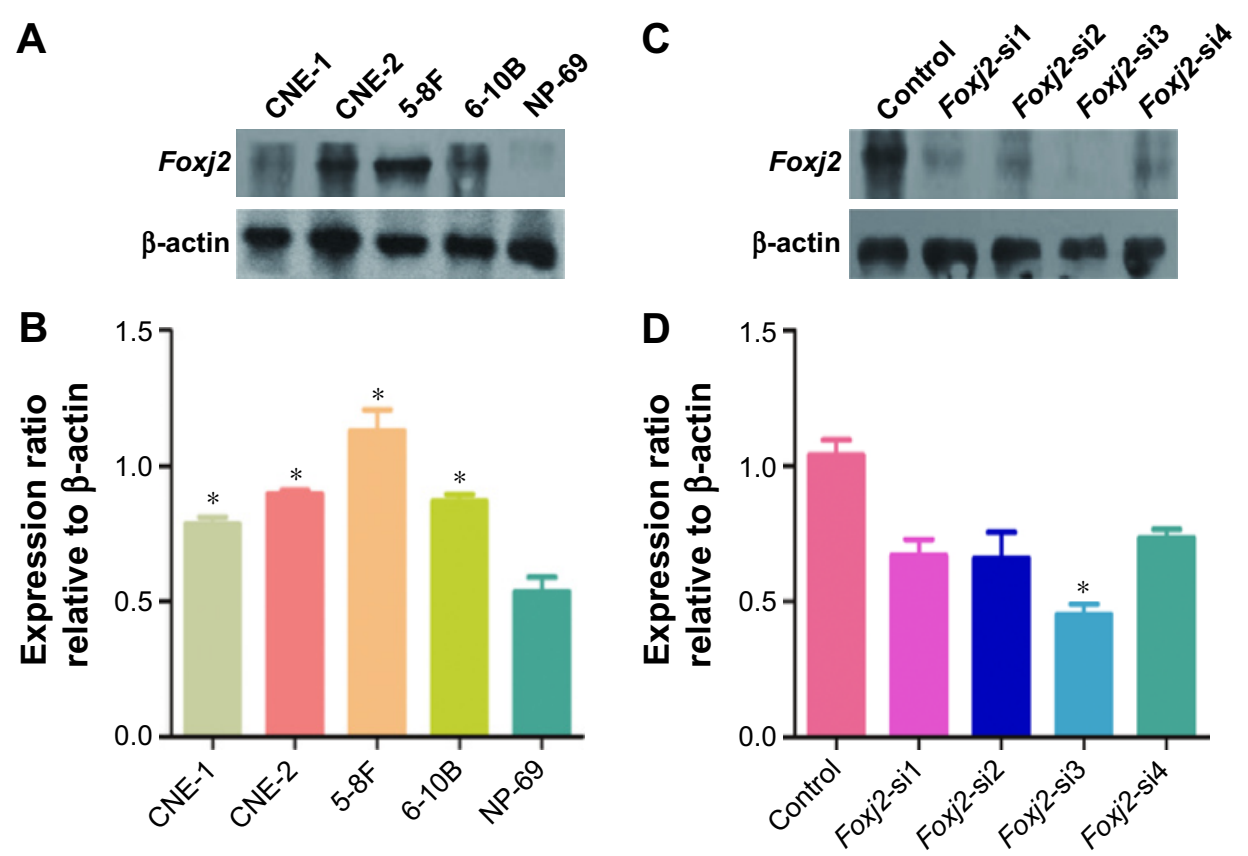

Figure 3 Expression of Foxj2 in NPC cells.

Notes: (A, B) Western blot analysis of Foxj2 expression in 4 kinds of NPC cell lines (CNE-I, CNE-2, 5-8F, and 6-10B) and in NP-69 cell line. (C, D) The expression of Foxj2 was suppressed using siRNAs, and interference efficiency was detected by Western blotting. $\beta$-actin was used as control. $* P<0.05$. Data are presented as mean \pm SD. Abbreviations: NPC, nasopharyngeal carcinoma; siRNA, small interfering RNA.

Foxj2 belongs to the human Forkhead box (Fox) family of proteins, which are implicated in carcinogenesis through their action of transcriptional regulation. ${ }^{28,29}$ Upregulation of these genes, caused by various mechanisms such as mutation, amplification, and gene fusion, leads to congenital disorders, diabetes mellitus, or carcinogenesis. ${ }^{30}$ Many Fox family genes, such as FoxA, FoxQ, FoxC, FoxM, and
Fox $O$, have been shown to play an important role in the genesis, progression, and cell dissemination of certain cancers. ${ }^{12-17,31,32}$ Previous evidences suggest that Foxj2 is abnormally expressed in various malignancies and is involved in positively regulating the progression of the cell cycle or actively participating in the metastatic process. ${ }^{20}$ The current study extended these findings by investigating
A
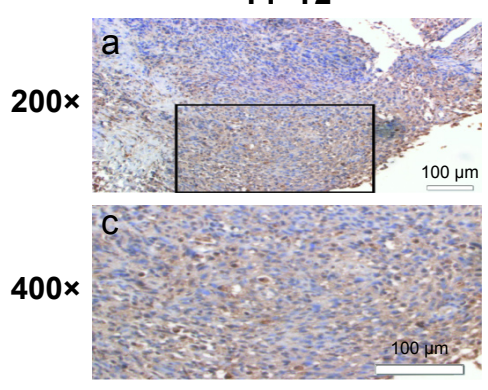

B

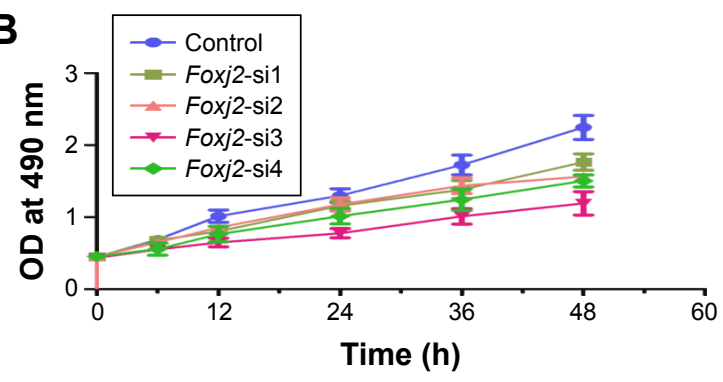

T3-T4

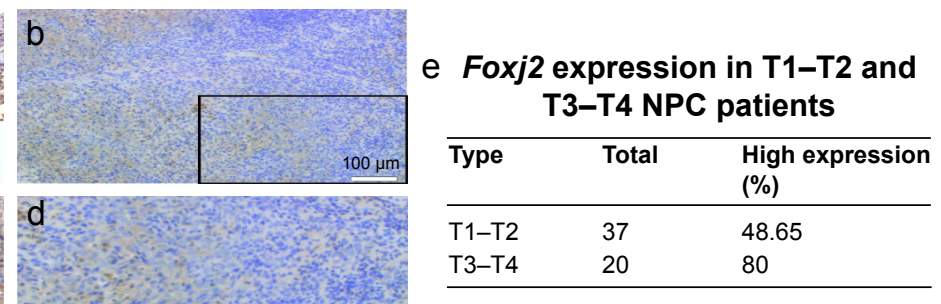

C

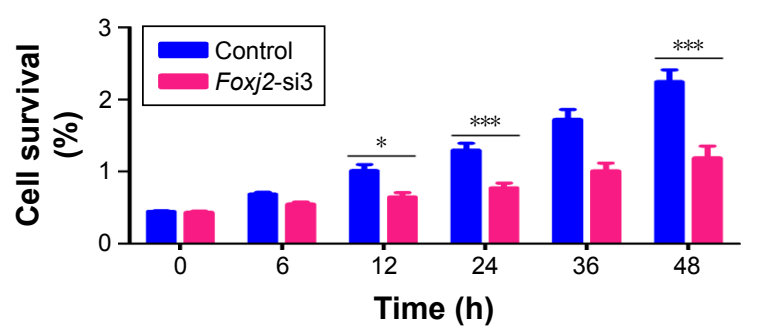

Figure 4 (Continued) 
D

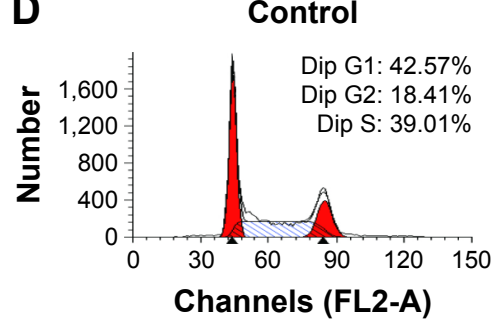

Foxj2-si3

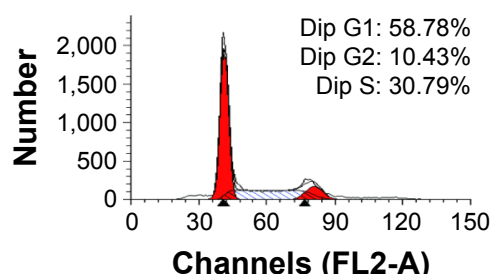

Channels (FL2-A)

\section{Foxj2-si1}

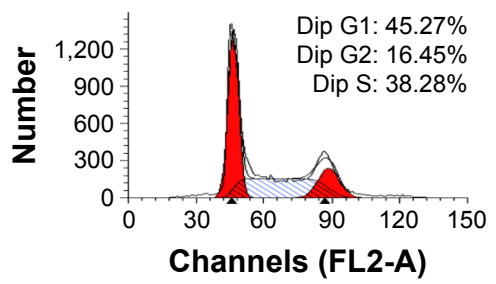

Foxj2-si4

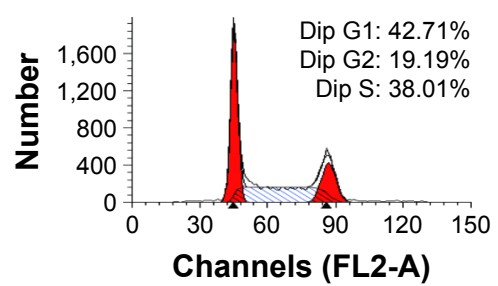

Channels (FL2-A)

$\square$ Dip G1 $\square$ Dip G2 Dip S
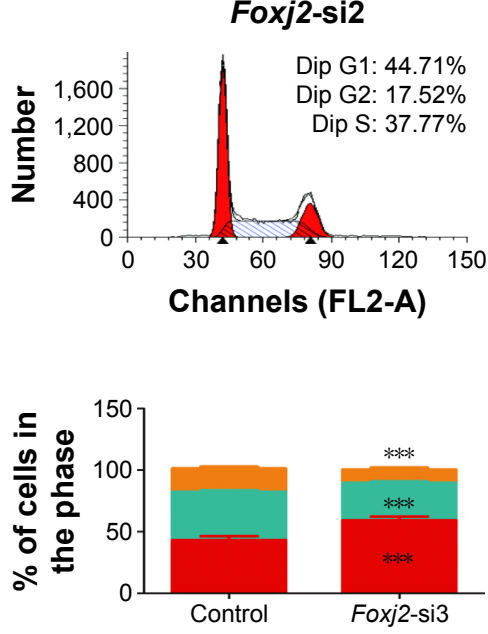

- G1 $-\mathrm{S}=\mathrm{G} 2$

Figure 4 Knockdown of Foxj2 reduces CNE-2 cell proliferation.

Notes: (A) IHC analysis (I:50, ab22857) of Foxj2 expression in TI-T2 and T3-T4 NPC tissues. Original magnifications: $\times 200$ (a-b); $\times 400$ (c-d); (e) percentage expression of Foxj2. (B, C) CCK-8 assay was used to determine cell proliferation of CNE-2 cells treated with Foxj2 siRNA for the indicated time. (D) Cell cycle distribution analyzed by flow cytometry in CNE-2 cells after Foxj2 downregulation. The data are presented as mean value \pm SEM (compared with the control). The same experiment was repeated at least 3 times. $* P<0.05 ; * * * P<0.001$. Data are presented as mean \pm SD.

Abbreviations: CCK-8, cell counting kit 8; IHC, immunohistochemistry; NPC, nasopharyngeal carcinoma; OD, optical density; siRNA, small interfering RNA.

Foxj2 expression and functions in NPC tissues and cell lines. We found that Foxj 2 was remarkably increased in NPC by IHC and Western blot. The association of Foxj2 expression with NPC clinicopathologic variables was further explored. As shown, overexpressed Foxj 2 was significantly associated with T-classification, distant metastasis, and clinical stage. These results indicated that the overexpressed Foxj2 might play an important role in NPC progression and migration. Several Fox family genes have been reported in the literature to influence NPC patients' prognosis. For example, Huang et $\mathrm{al}^{33}$ recently observed that elevated expression of FoxM1 predicts poor survival in patients with NPC. Our research confirmed that patients with Foxj2 overexpression had a shorter survival time and overexpressed Foxj2 was a remarkable independent predictor of poor prognosis for NPC. Therefore, Foxj2 expression in NPC might serve as a predictor for the clinical outcome.

Chen et $\mathrm{al}^{34}$ established that Foxj2 was colocalized with proliferating cell nuclear antigen (PCNA), which had been used as a general marker of cell proliferative activity. However, the contribution of Foxj 2 to malignant proliferation of cancers including NPC remained largely unidentified and need to be determined. In order to explore this role, a series of experiments were performed in cell lines. We
A
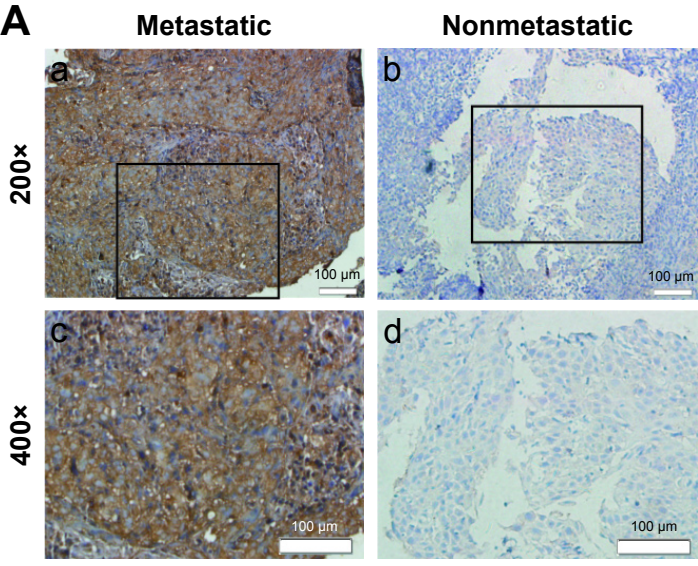

e Foxj2 expression in metastatic and nonmetastatic NPC patients

\begin{tabular}{lll}
\hline Type & Total & $\begin{array}{l}\text { High } \\
\text { expression (\%) }\end{array}$ \\
\hline Metastatic & 14 & 92.86 \\
Nonmetastatic & 43 & 48.84 \\
\hline
\end{tabular}

Figure 5 (Continued) 

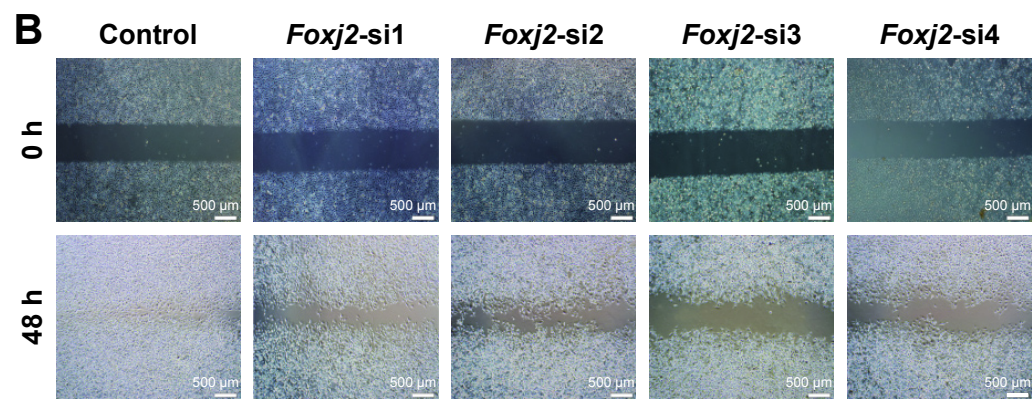

D

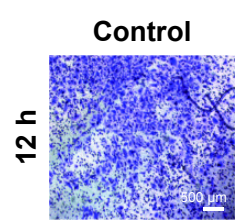

Foxj2-si1

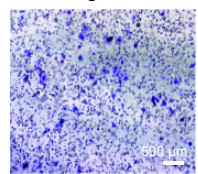

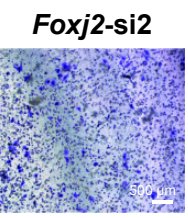
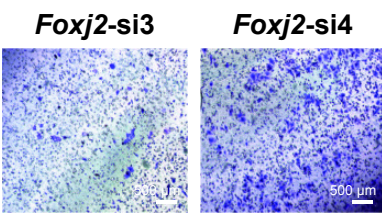

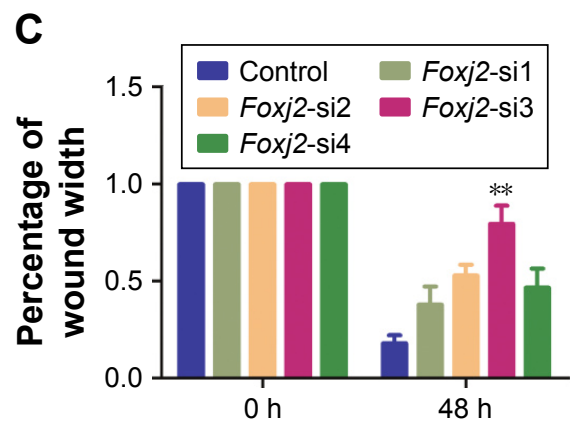

E

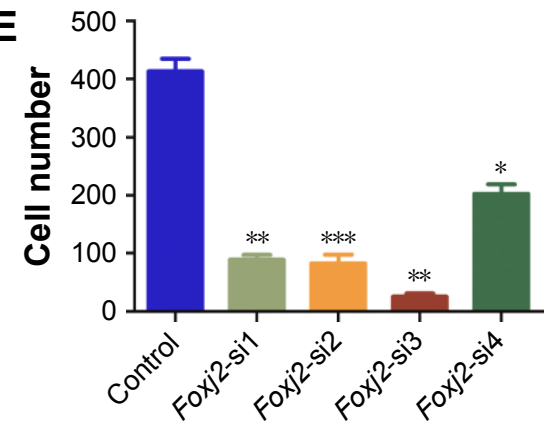

Figure 5 Knockdown of Foxj2 decreases the migration of CNE-2 cells.

Notes: (A) IHC analysis ( I:50, ab22857) of Foxj2 expression in metastatic and nonmetastatic NPC tissues. Original magnifications: $\times 200$ (a-b); $\times 400$ (c-d); (e) percentage expression of Foxj2. (B) CNE-2 cells transfected with Foxj2 siRNAs showed a slower migration rate than those transfected with the control siRNA. Migration of cells to the wound was visualized at 0 and $48 \mathrm{~h}$ with a microscope (original magnification: $\times 200$ ). (C) The histogram shows the relative migration distance of (B). (D-E) Knockdown of Foxj2 in CNE-2 cells inhibited cell migration, as determined by transwell assays. Number of cells migrating through the membrane was counted in 10 fields under $\times 20$ objective lens. Error bars, SD. $n=3 ; * P<0.05, * * p<0.01, * * * p<0.001$. The same experiment was repeated at least 3 times.

Abbreviations: IHC, immunohistochemistry; NPC, nasopharyngeal carcinoma; siRNA, small interfering RNA.

used siRNA to knock down Foxj2 expression in NPC cells. After Foxj2 was silenced, we found that the proliferation of CNE-2 cells was significantly inhibited. Cell cycle analysis demonstrated that downregulation of Foxj2 decreased the cell population in the $\mathrm{S}$ phase as well as augmented G1 cycle arrest in CNE-2 cells, leading to inability to complete cell division. As Foxj2 participates in regulating the cell cycle, this might partly explain the mechanism by which Foxj2 accelerated cell proliferation. Taken together, these findings indicated that Foxj 2 might contribute to carcinogenesis by regulating cell proliferation through the regulation of cell cycle distribution.

Increasing evidence suggests that Foxj2 might actively participate in the metastatic process of various cancers, including glioma, extrahepatic cholangiocarcinoma, breast cancer, and lung cancer, ${ }^{21,22,24,35}$ as NPC patients with metastasis had a higher rate of treatment failure and mortality. ${ }^{26}$ As NPC has the characteristics of highly malignant local invasion and early distant metastasis and metastasis is an important character that influences NPC patients' prognosis, we tried to explore the relationship between Foxj2 and metastasis in NPC. Wound-healing and transwell migration assays demonstrated that silencing of Foxj 2 inhibited the migration of CNE- 2 cells. These data suggested that Foxj 2 overexpression promoted metastasis of NPC.

\section{Conclusion}

This study indicated that Foxj2 functioned as a potential oncogene and played an important role in NPC progression and migration. In this research, we confirmed that Foxj 2 was upregulated in NPC and the level of Foxj2 is associated with the clinical progression and poor prognosis. Furthermore, our data obtained by downregulation of Foxj2 expression establish the role of Foxj2 in modulating the biological properties of NPC cells, including proliferation and migration. We believe that precise understanding of the biological functions of Foxj2 will provide specific targets for cancer therapeutic intervention.

\section{Acknowledgment}

This study was funded by the Chinese National Natural Science Foundation (grant numbers 81672682 and 81602385 ) and the Natural Science Foundation of Jiangsu Province (grant number SBK2015022581). 


\section{Disclosure}

The authors report no conflicts of interest in this work.

\section{References}

1. Petersson F. Nasopharyngeal carcinoma: a review. Semin Diagn Pathol. 2015;32(1):54-73.

2. Leung TW, Tung SY, Sze WK, et al. Treatment results of 1070 patients with nasopharyngeal carcinoma: an analysis of survival and failure patterns. Head Neck. 2005;27(7):555-565.

3. Hannenhalli S, Kaestner KH. The evolution of Fox genes and their role in development and disease. Nat Rev Genet. 2009;10(4):233-240.

4. Lehmann OJ, Sowden JC, Carlsson P, Jordan T, Bhattacharya SS. Fox's in development and disease. Trends Genet. 2003;19(6):339-344.

5. Zhou S, Zawel L, Lengauer C, Kinzler KW, Vogelstein B. Characterization of human FAST-1, a TGF beta and activin signal transducer. Mol Cell. 1998;2(1):121-127.

6. Kaestner KH, Silberg DG, Traber PG, Schutz G. The mesenchymal winged helix transcription factor Fkh6 is required for the control of gastrointestinal proliferation and differentiation. Genes Dev. 1997; 11(12):1583-1595.

7. Kume T, Deng KY, Winfrey V, Gould DB, Walter MA, Hogan BL. The forkhead/winged helix gene Mf1 is disrupted in the pleiotropic mouse mutation congenital hydrocephalus. Cell. 1998;93(6):985-996.

8. Lin K, Dorman JB, Rodan A, Kenyon C. Daf-16: an HNF-3/forkhead family member that can function to double the life-span of Caenorhabditis elegans. Science. 1997;278(5341):1319-1322.

9. Ogg S, Paradis S, Gottlieb S, et al. The fork head transcription factor DAF-16 transduces insulin-like metabolic and longevity signals in C. elegans. Nature. 1997;389(6654):994-999.

10. Myatt SS, Lam EW. The emerging roles of forkhead box (Fox) proteins in cancer. Nat Rev Cancer. 2007;7(11):847-859.

11. Benayoun BA, Caburet S, Veitia RA. Forkhead transcription factors: key players in health and disease. Trends Genet. 2011;27(6):224-232.

12. Yu C, Chen L, Yie L, et al. Targeting FoxM1 inhibits proliferation, invasion and migration of nasopharyngeal carcinoma through the epithelial-to-mesenchymal transition pathway. Oncol Rep. 2015;33(5): 2402-2410.

13. Wang W, Yi M, Chen S, et al. Significance of the NOR1-FOXA1/ HDAC2-slug regulatory network in epithelial-mesenchymal transition of tumor cells. Oncotarget. 2016;7(13):16745-16759.

14. Peng XH, Huang HR, Lu J, et al. MiR-124 suppresses tumor growth and metastasis by targeting Foxq1 in nasopharyngeal carcinoma. Mol Cancer. 2014;13:186.

15. Li Y, Chen X. MiR-4792 inhibits epithelial-mesenchymal transition and invasion in nasopharyngeal carcinoma by targeting FOXC1. Biochem Biophys Res Commun. 2015;468(4):863-869.

16. Ou-Yang L, Xiao SJ, Liu P, et al. Forkhead box C1 induces epithelialmesenchymal transition and is a potential therapeutic target in nasopharyngeal carcinoma. Mol Med Rep. 2015;12(6):8003-8009.

17. Zhou Z, Zhang L, Xie B, et al. FOXC2 promotes chemoresistance in nasopharyngeal carcinomas via induction of epithelial mesenchymal transition. Cancer Lett. 2015;363(2):137-145.
18. Perez-Sanchez C, Gomez-Ferreria MA, de La Fuente CA, et al. FHX, a novel fork head factor with a dual DNA binding specificity. $J$ Biol Chem. 2000;275(17):12909-12916.

19. Granadino B, Arias-de-la-Fuente C, Perez-Sanchez C, et al. Fhx (Foxj2) expression is activated during spermatogenesis and very early in embryonic development. Mech Dev. 2000;97(1-2):157-160.

20. Kehn K, Berro R, Alhaj A, et al. Functional consequences of cyclin D1/BRCA1 interaction in breast cancer cells. Oncogene. 2007;26(35): 5060-5069.

21. Wang Y, Yang S, Ni Q, et al. Overexpression of forkhead box J2 can decrease the migration of breast cancer cells. J Cell Biochem. 2012; 113(8):2729-2737.

22. Qiang Y, Wang F, Yan S, et al. Abnormal expression of forkhead box J2 (FOXJ2) suppresses migration and invasion in extrahepatic cholangiocarcinoma and is associated with prognosis. Int J Oncol. 2015; 46(6):2449-2458.

23. Yang Q, Cao X, Tao G, et al. Effects of FOXJ2 on TGF-beta1-induced epithelial-mesenchymal transition through Notch signaling pathway in non-small lung cancer. Cell Biol Int. 2017;41(1):79-83.

24. Qiu X, Ji B, Yang L, et al. The role of FoxJ2 in the migration of human glioma cells. Pathol Res Pract. 2015;211(5):389-397.

25. You Y, Yao H, You B, et al. Clinical significance of HAX-1 expression in laryngeal carcinoma. Auris Nasus Larynx. 2015;42(4):299-304.

26. Shi S, Cao X, Gu M, You B, Shan Y, You Y. Upregulated expression of SOX4 is associated with tumor growth and metastasis in nasopharyngeal carcinoma. Dis Markers. 2015;2015:658141.

27. You B, Shan Y, Shi S, Li X, You Y. Effects of ADAM10 upregulation on progression, migration, and prognosis of nasopharyngeal carcinoma. Cancer Sci. 2015;106(11):1506-1514.

28. Coffer PJ, Burgering BM. Forkhead-box transcription factors and their role in the immune system. Nat Rev Immunol. 2004;4(11):889-899.

29. Perez-SanchezC,Arias-de-la-Fuente C, Gomez-Ferreria MA, GranadinoB, Rey-Campos J. FHX.L and FHX.S, two isoforms of the human forkhead factor FHX (FOXJ2) with differential activity. $J$ Mol Biol. 2000;301(4):795-806.

30. Katoh M, Katoh M. Human FOX gene family (review). Int J Oncol. 2004;25(5):1495-1500.

31. Du J, Li L, Ou Z, et al. FOXC1, a target of polycomb, inhibits metastasis of breast cancer cells. Breast Cancer Res Treat. 2012;131(1):65-73.

32. Lou K, Chen N, Li Z, et al. MicroRNA-142-5p overexpression inhibits cell growth and induces apoptosis by regulating FOXO in hepatocellular carcinoma cells. Oncol Res. 2017;25(1):65-73.

33. Huang PY, Li Y, Luo DH, et al. Expression of aurora-B and FOXM1 predict poor survival in patients with nasopharyngeal carcinoma. Strahlenther Onkol. 2015;191(8):649-655.

34. Chen X, Cao X, Tao G, et al. FOXJ2 expression in rat spinal cord after injury and its role in inflammation. J Mol Neurosci. 2012; 47(1):158-165.

35. Dubey R, Saini N. STAT6 silencing up-regulates cholesterol synthesis via miR-197/FOXJ2 axis and induces ER stress-mediated apoptosis in lung cancer cells. Biochim Biophys Acta. 2015;1849(1):32-43.
OncoTargets and Therapy

\section{Publish your work in this journal}

OncoTargets and Therapy is an international, peer-reviewed, open access journal focusing on the pathological basis of all cancers, potential targets for therapy and treatment protocols employed to improve the management of cancer patients. The journal also focuses on the impact of management programs and new therapeutic agents and protocols on

Submit your manuscript here: http://www.dovepress.com/oncotargets-and-therapy-journal

\section{Dovepress}

patient perspectives such as quality of life, adherence and satisfaction. The manuscript management system is completely online and includes a very quick and fair peer-review system, which is all easy to use. Visit http://www.dovepress.com/testimonials.php to read real quotes from published authors. 